\title{
Dispatching and Optimal Operation of Power Grid Based on Smart Grid Dispatching
}

\author{
Denghui Cheng \\ College of Mechanical Engineering ,Sichuan Agricultural University, Yaan, 625014 ,Sichuan Province , \\ 625014, China
}

Keywords: Operation; power grid; Smart Grid; Dispatching

\begin{abstract}
In view of the current situation of large-scale centralized development of new energy sources in China, the necessity of developing new energy dispatching and the characteristics of new energy dispatching are analyzed. In view of the traditional scheduling technology support system has been unable to cope with large-scale new energy grid to grid dispatching operation challenges, introduces a new energy coordination optimization scheduling method based on multi time scale, including the annual / monthly consumption of new energy plan, and before the days of rolling scheduling, and scheduling is based on smart grid the application of control system design platform, new energy technology support system. This system can realize the coordinated scheduling of multiple time scale of the new energy, gradually reduce the prediction error of new energy, to ensure maximum safety of new energy consumption. The research results have been applied in 16 national grid / provincial power grids.
\end{abstract}

\section{Introduction}

With the global energy crisis increasingly serious, the social needs of environmental protection, electricity market steadily, the user's demand for power services continues to improve, the user urgently needs a more stable, safer, more economical, more efficient, more environmentally friendly power. Therefore, the concept of smart grid arises at the historic moment, and has become a research hotspot of the future grid in recent years. Smart grid provides a high operating efficiency, reliability and cost reduction blueprint for related enterprises. Through the production, transportation, retail and other aspects of management, you can save electricity, realize intelligent management, improve renewable energy utilization, support hybrid electric vehicles access, and home appliances intelligent. Power Grid Corp mainly considers the operation safety, reliability and economy of power grid. Electricity users mainly consider electricity expenditure and stable power supply. Therefore, improve power system security, reliability and economy, reduce electricity costs, improve energy efficiency, to achieve energy conservation, low-carbon environmental protection, is the fundamental goal of smart grid construction project in the wind and solar (PV) as the main research object, to meet the trend of the times and the needs of development, is very important the practical significance and use value. Power grid is a very complex system, as one of the most classic civilizations in human history, its value and people's demand for it can be imagined. The project focuses on the optimal scheduling model of wind power and photovoltaic power generation. Electrical energy from the beginning of the power station from the power generation and transmission of electricity and other links. Power grid dispatching is one of the most important links. The appropriate scheduling scheme can effectively reduce the energy consumption, greatly increase the economic and social benefits, but also improve the power quality and safety of its users.

\section{Research Status Analysis}

Wind power and photovoltaic power generation technology is becoming more and more mature, and the future smart distribution network must be compatible with new energy, realize the interaction of load side, support the flexible access and convenient use of diversified power supply. 
However, our country is currently in the initial stage of the rational scheduling of new energy optimization is still in the initial stage, so it needs more investment. Meanwhile, the large-scale grid connection of new energy brings difficulties and challenges to the dispatching operation of power grid. On the one hand, the new energy output forecast precision is low, the scheduling plan and actual operation of large deviation to schedule adjustment even can not balance the actual power; on the other hand, the fluctuation of new energy inherent, makes the regulating capacity, FM capacity of power system and reserve capacity is difficult to determine accurately, easy to cause the abandoned wind abandon, light, load shedding phenomenon. Therefore, the dispatching model is suitable for large scale research for the new energy, to achieve a variety of strong random power real-time power balance of power system, to reduce and avoid the abandoned wind, abandoned light, load shedding and other phenomena, large-scale development of new energy and power to promote, has important significance.

\section{Method Implementation}

Hadoop platform is a distributed data processing platform with high stability and good scalability. It is the most widely used open source cloud computing software platform. The existing task scheduling algorithms of Hadoop platform mainly include three algorithms: FIFO scheduling algorithm, computing power scheduling algorithm and fair scheduling algorithm. Fair scheduling algorithm is used to ensure large and small tasks can be quickly response, but also to ensure the service level of the task, the purpose is to provide equal resources for each task. If there is only one task in the cluster, the task can enjoy the whole cluster resource. When there are other tasks submitted, a part of the resource space will be available for the new task service, fair scheduling algorithm is used to organize the resource pool, a fair share of the resources in the resource pool, each user by default only a pool of resources, even if each user submits the number of tasks is not the same, a the fair resource sharing the same way. The fair scheduler allows the allocation of the minimum guaranteed shared resources for the resource pool, which ensures that a specific user can get enough shared resources. When each resource pool containing tasks needs less than the minimum guaranteed shared resource, the additional part will be segmented by other resource pools. Fair scheduling algorithm allows the task to run all by default, the number of tasks can also change the configuration file to limit each user and each resource pool can run, because at the same time to deal with a large number of tasks will produce a large amount of intermediate data, seriously affect the performance of the system. The limit of the maximum number of tasks is not the task that refuses to be submitted, but the task is temporarily put into the queue for waiting, and then the task to be run is selected according to the priority of each task and the submission time.

This project is based on the knowledge system of power system, and focuses on the research of new energy grid dispatching mode. In this project, we take wind power generation and photovoltaic power generation scheduling as the main object of study, respectively for the comprehensive analysis of the two, and the use of mathematical modeling, power system simulation, probability distribution algorithm to explore it. This paper consists of five modules: generation characteristics and output probability research, wind light combined output power probability research, suitable algorithm solution model, establishment of reasonable mathematical optimization wind power scheduling mathematical model, results analysis and model evaluation promotion. The following is the specific implementation of the process plan.

According to the characteristics of the smart grid dispatching system and the feasibility of the application of the "intelligent power cloud" information platform, a smart grid dispatching system based on cloud computing is proposed. The application of cloud computing technology, the distributed data service bus system, in a structured way to disperse the IT data information resources and network automation infrastructure together with smart grid scheduling cloud of high reliability, high real-time, high accuracy and computing platform for output probability research module generating characteristics research, four aspects including the module, respectively is the study of characteristics of wind power, wind power output probability distribution, photovoltaic power generation, photovoltaic power generation characteristics of the probability distribution of 
the four directions.

Cloud computing grid scheduling system is a flexible network topology of large-scale distributed network architecture, distributed data service bus system to support one to one, one to many, many to one and many to many connection types, structure of each sub scheduling platform can be formed with tree type, bus type and star type, are multistage dynamic scheduling also among each dispatching platform; bus can also integrate the sub scheduling platform server, the underlying business resources and low latency data transmission between nodes sharing capabilities, the system provides the guarantee for the continuous availability; distributed data service bus also supports to modify the configuration parameters, adding dynamic application agreement, expansion and so on, and can ensure the automatic recovery in the process of node failure and after the failure of the system; the efficient remote management function and powerful The log function is convenient for dispatchers to adjust the system parameters and monitor the network status, and provide reliable guarantee for the search and management system. For the wind light combined output power probability module, the module is mainly based on the previous module, and a simple mathematical model is established to predict the probability of the common output power of the two new energy sources. To establish a reasonable mathematical optimization for wind - light power module scheduling mathematical model, the first module to calculate power prediction interval, the process needs to set up the objective function and the constraint condition and the model is linearized, and then using time series method and differential equation to predict the power output of the wind light power combination forecasting method and the. For the appropriate module, the model adopts a variety of mathematical models, such as adaptive robust optimization method, robust scheduling method with affine rectification process and robust optimization method with maximum acceptable perturbation range. On the basis of the worst environment, the acceptable scheduling method is calculated. Advanced optimization algorithms can quickly find the solution of these models to solve the model.

The results of analysis and evaluation model module, the module to get the optimal scheduling results and analysis the advantages and disadvantages of the model, then set up the basic system parameters of power system simulation software (such as: PSAPAC, NETOMAC, PSS/E, OPF simulation software, as shown in Figure 1) generator simulation model, and then the basic application of the model, and analysis feasibility and scientific application in practice.

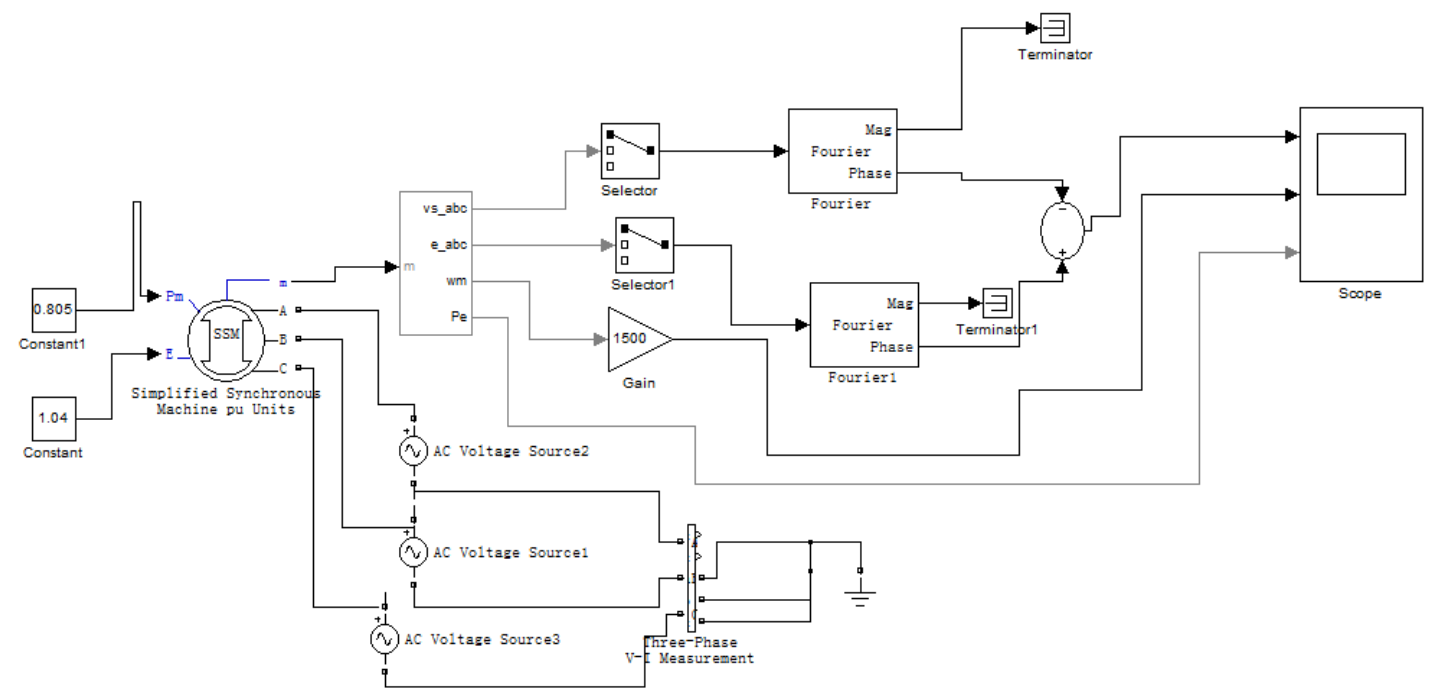

Fig. 1 generator simulation model

\section{Summary}

With the support of the government and the advantages of smart grid itself, more and more researchers have devoted themselves to the research of smart grid technology. Although a large 
number of literatures have studied the economic dispatch of smart grid and renewable resources power generation, there are few studies on the economic dispatch strategy of Smart Grid Considering Flexible load. In addition, the application of multi-agent based distributed algorithms to the design and innovation of smart grid is also a research point. Therefore, this paper carried out the following work: the smart grid is introduced, including the concept of smart grid, the development status at home and abroad, and the key technologies of smart grid are expounded; it also introduces the application of multi-agent technology in intelligent power grid. Considering the flexible load access to the smart grid, the meaning of the economic dispatch in the power system has changed, that is, the generator and flexible load can maximize the social welfare of the whole power system under certain restrictions.

\section{References}

[1] Weisen Pan, Shizhan Chen, ZhiyongFeng. Investigating the Collaborative Intention and Semantic Structure among Co-occurring Tags using Graph Theory. 2012 International Enterprise Distributed Object Computing Conference, IEEE, Beijing, pp. 190-195.

[2] Jennifer W. Chan, Yingyue Zhang, and Kathryn E. Uhrich, Amphiphilic Macromolecule Self-Assembled Monolayers Suppress Smooth Muscle Cell Proliferation, Bioconjugate Chemistry, 2015, 26(7), 1359-1369.

[3] Yingyue Zhang, Evan Mintzer, and Kathryn E. Uhrich, Synthesis and Characterization of PEGylatedBolaamphiphiles with Enhanced Retention in Liposomes, Journal of Colloid and Interface Science, 2016, 482, 19-26.

[4] Jonathan J. Faig, AlyshaMoretti, Laurie B. Joseph, Yingyue Zhang, Mary Joy Nova, Kervin Smith, and Kathryn E. Uhrich, Biodegradable Kojic Acid-Based Polymers: Controlled Delivery of Bioactives for Melanogenesis Inhibition, Biomacromolecules, 2017, 18(2), 363-373.

[5] Lv, Z., Halawani, A., Feng, S., Li, H., \&Réhman, S. U. (2014). Multimodal hand and foot gesture interaction for handheld devices. ACM Transactions on Multimedia Computing, Communications, and Applications (TOMM), 11(1s), 10. 